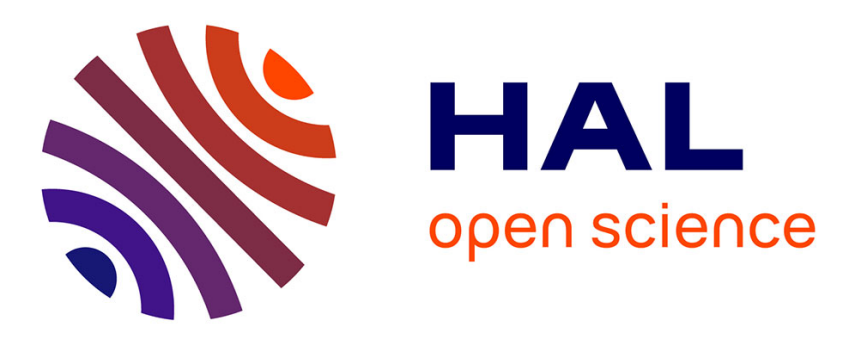

\title{
L'exploration du cryokarst glaciaire et son intérêt scientifique pour l'étude du drainage des eaux de fonte: porches, cavités, crevasses, bédières et moulins
}

Luc Moreau

\section{- To cite this version: \\ Luc Moreau. L'exploration du cryokarst glaciaire et son intérêt scientifique pour l'étude du drainage des eaux de fonte: porches, cavités, crevasses, bédières et moulins. Neige et glace de montagne: Reconstitution, dynamique, pratiques, Collection EDYTEM - Cahiers de Géographie, n8, pp.163- 170, 2009. halsde-00404048}

\section{HAL Id: halsde-00404048 \\ https://hal.science/halsde-00404048}

Submitted on 31 Mar 2011

HAL is a multi-disciplinary open access archive for the deposit and dissemination of scientific research documents, whether they are published or not. The documents may come from teaching and research institutions in France or abroad, or from public or private research centers.
L'archive ouverte pluridisciplinaire HAL, est destinée au dépôt et à la diffusion de documents scientifiques de niveau recherche, publiés ou non, émanant des établissements d'enseignement et de recherche français ou étrangers, des laboratoires publics ou privés. 


\section{Collection EDYTEM}

Numéro 8 - Année 2009

Cahiers de

Géographie

\section{NEIGE et GLACE de MONTAGNE}

Reconstitution, dynamique, pratiques

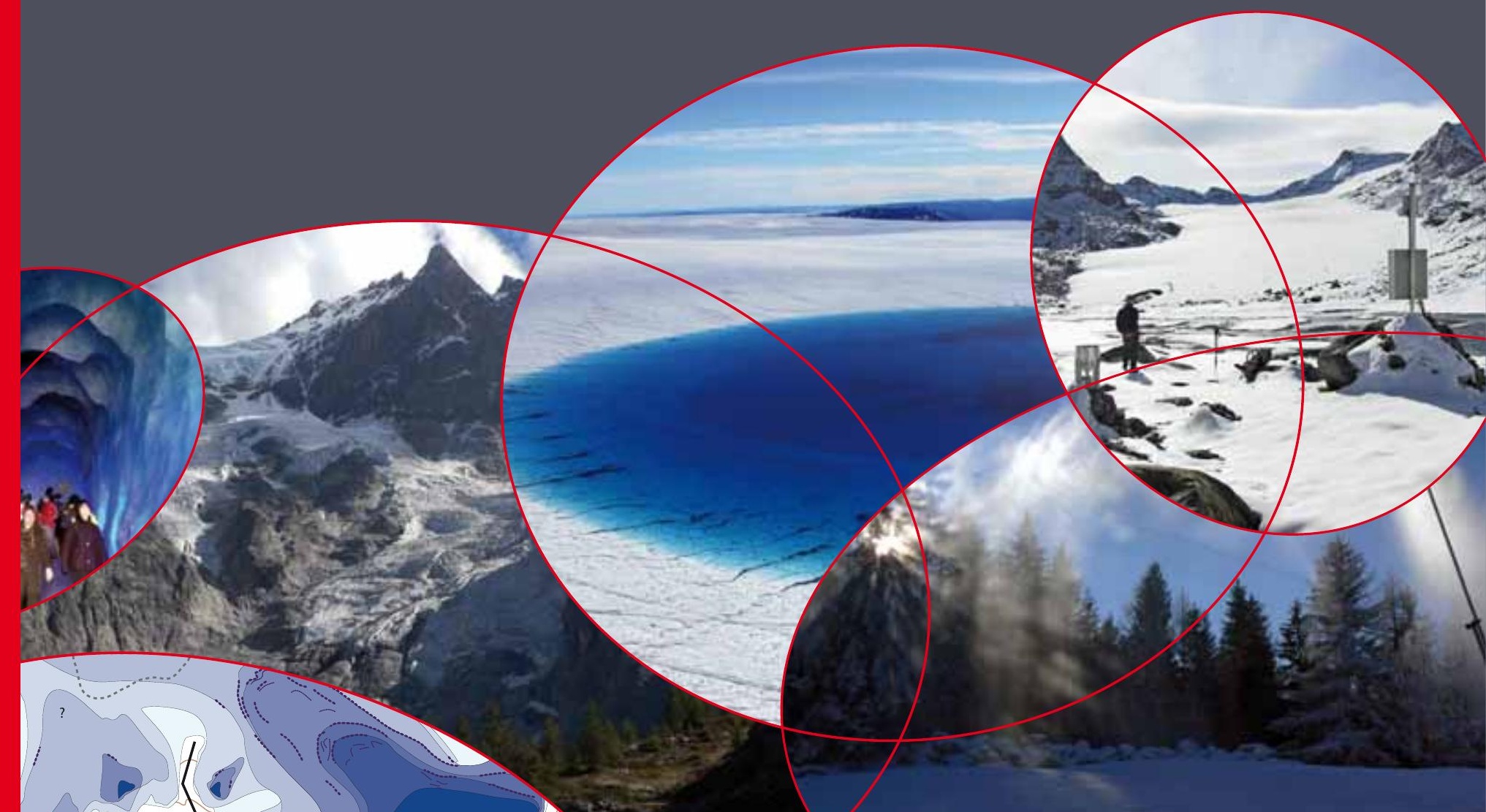





\title{
L'EXPLORATION DU CRYOKARST GLACIAIRE ET SON INTÉRÊT SCIENTIFIQUE POUR L'ÉTUDE DU DRAINAGE DES EAUX DE FONTE
}

\section{PORCHES, CAVITÉS, CREVASSES, BÉDIÈRES ET MOULINS}

\author{
GLACIAL CRYOKARST EXPLORATION AND HIS SCIENTIFIC INTEREST \\ IN THE STUDY OF MELT WATER DRAINAGE: \\ GLACIAL MOUTHS, CAVITIES, CREVASSES, BEDIERES AND MOULINS
}

LUC MOREAU

Laboratoire EDYTEM, Université de Savoie/CNRS, Campus scientifique, F 73376 Le Bourget-du-Lac cedex.

Contact : moreauluc@club-internet.fr

\begin{abstract}
RÉSUMÉ
Il est important de connaître les conditions de circulation des eaux dans les niveaux supra-, intra- et sous-glaciaire, car leur rôle est primordial pour l'écoulement du glacier, l'érosion de gorge ou de vallée, la déformation du till sous-glaciaire, et la formation des poches d'eau ou des lacs. Pourtant, les niveaux intraglaciaire et sous-glaciaire restent encore mal connus, car l'accès y est difficile et très localisé.

Nous présentons brièvement les différents accès naturels qui permettent de pénétrer les glaciers, en particulier les moulins, dont l'exploration nous renseigne sur les conditions de drainage qui contrôlent le glissement des glaciers - avec une accélération l'été dans les Alpes, mais également au Groenland depuis quelques années. Les crevasses marginales qui favorisent la circulation de l'eau vers les marges et la contribution possible de celle-ci dans le creusement des vallées en auges sont évoquées.
\end{abstract}

Mots-CLÉS : HYDRO-GLACIOLOGIE, CRYOKARST, BÉDIÈRE, MOULIN, ALPES, GROENLAND.

\begin{abstract}
Drainage conditions of the water in the different levels of the glaciers are important, because of its control on ice flow subglacial erosion of grooves or valleys, subglacial till deformation, and formation of water pockets or lakes. But intra-and subglacial levels are still poorly known, because the access is difficult and very local.

We present the different natural access to enter the glaciers. Moulins are probably the most suitable to document drainage conditions that control the sliding of glaciers and its acceleration observed in summer in the Alps, and in Greenland since a few years. The role in terms of erosion of marginal crevasses that drain water towards the margins of the glacier, and its contribution to the trough erosion, are emphasized here.
\end{abstract}

KEYWORDS: HYDROGLACIOLOGY, CRYOKARST, BEDIERE, MOULIN, ALPS, GREENLAND. 
"Quelle chance auront nos successeurs...

peut-être un jour de découvrir le monde sous-glaciaire et tous ses mystères. »

(Louis Agassiz, 1807-1873)

\section{INTRODUCTION}

Le cryokarst est l'ensemble des cavités naturelles supra-, intra- ou sous-glaciaires, chenaux, galeries, conduits, sculptés par les eaux ou l'air chaud, ou qui résultent de la topographie et de la déformation du glacier. Son rôle est primordial pour le drainage des eaux de surface vers la base du glacier. Il peut sembler paradoxal que le glacier, masse d'eau à l'état solide, soit parcouru en tout sens par l'eau liquide du printemps à l'automne, voire même en hiver. Cette eau a plusieurs origines :

- eau atmosphérique (pluie);

- eau de fusion de la neige, une fusion qui est totale jusqu'à la ligne d'équilibre glaciaire mais possible jusqu'à $3700 \mathrm{~m}$ d'altitude dans les Alpes, où elle imbibe le manteau neigeux et rend la glace tempérée (très proche du point de fusion) en fin d'été ;

- eau de fusion de la glace de surface (12-15 m de glace fondue l'été à la Mer de Glace au niveau du Montenvers ;

- eau de fusion de la glace basale, qui résulte du frottement et du flux géothermique ;

- eaux de fonte des versants, qui accèdent latéralement à la base du glacier ;

- sources sous-glaciaires enfin.
Dans les glaciers tempérés, ou qui vont le devenir avec le réchauffement climatique, l'eau liquide est donc présente en grande quantité, et ces glaciers sont à l'origine de rivières. Les eaux ont également un rôle très important dans la dynamique glaciaire et dans l'érosion du lit rocheux. Cette eau ne regèle pas dans le glacier tempéré et elle peut s'accumuler dans les crevasses ou sous forme de lacs ou de poches d'eau sous- ou intraglaciaires (glacier de Tête Rousse en 1892) dont les volumes ne permettent pas leur congélation totale.

L'été, l'eau de fonte s'écoule en petits ruisselets de surface sur la glace imperméable, puis dans des bédières, pour finalement se perdre dans des crevasses ou des moulins, ou alimenter un lac supraglaciaire (photo 1), un lac de confluence (glacier du Gorner) ou un lac juxtaglaciaire, à barrage morainique (lac du Miage) ou glaciaire (Marjelensee au glacier d'Aletsch). Dans le glacier, l'eau transite à travers la masse de glace à la faveur de fractures et de chenaux intraglaciaires avant de rejoindre par gravité le lit rocheux, puis de ressortir au front du glacier par un porche glaciaire. Pendant tout son parcours, l'eau a creusé et emprunté le cryokarst pour arriver à l'exutoire où elle devient rivière.

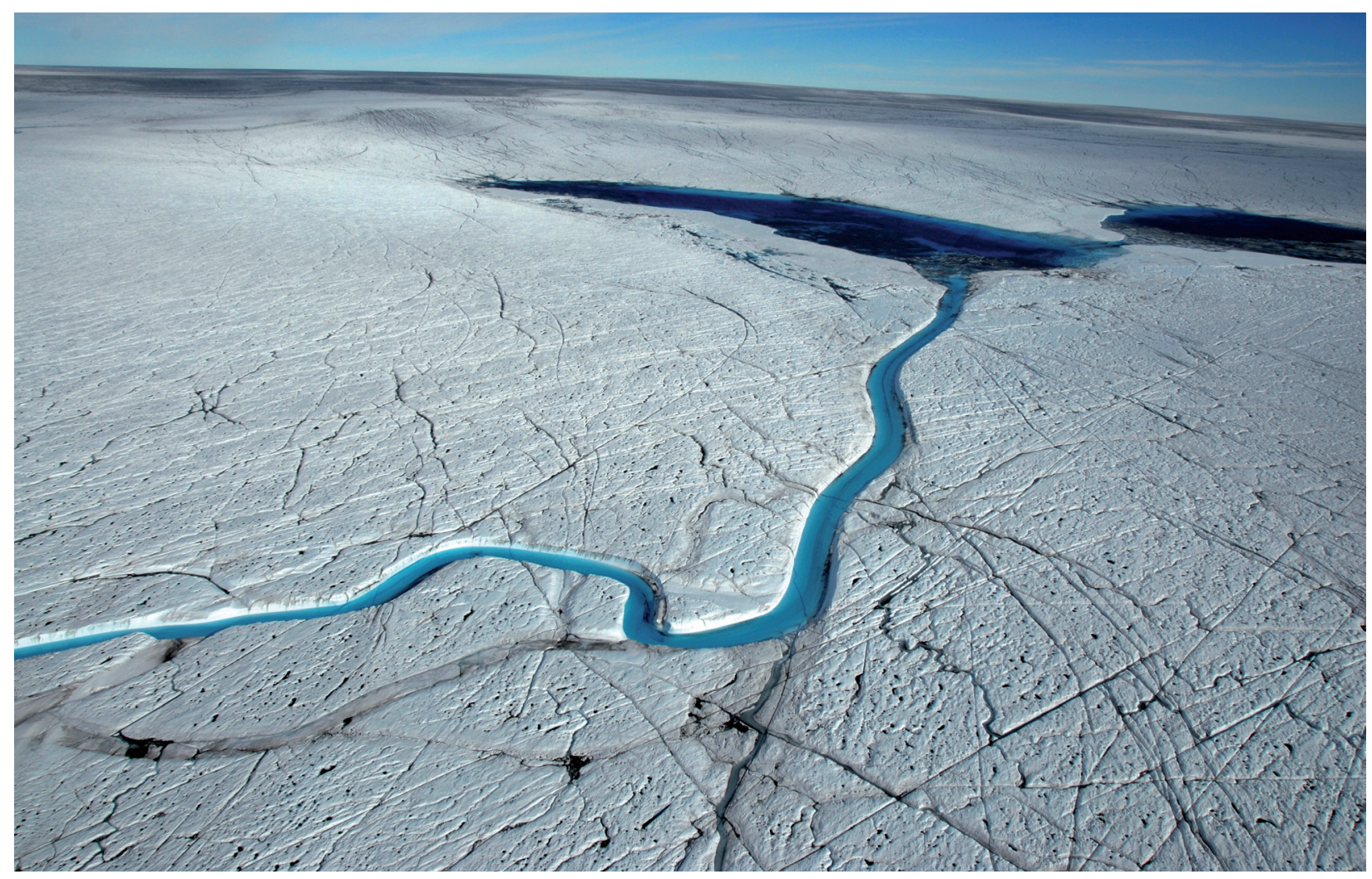

Photo 1 - Bédière et lacs supraglaciaires au nord du glacier d'Illulissat (Groenland, 07/2007). 


\section{I - LES CAVITÉS NATURELLES SUR LA MARGE DES GLACIERS}

\section{1 - Porches glaciaires :} les premières explorations

Ce sont certainement les porches (ou portails) glaciaires qui ont été les premières cavités visitées, du fait de leur facilité d'accès (figure 1). Sources de rivières, comme l'Arveyron au front du glacier des Bois (Mer de Glace), les porches grandioses sont longtemps restés des lieux touristiques, bien que parfois dangereux à cause d'une glace peu épaisse, fracturée, et des chutes de pierre fréquentes depuis la surface du glacier. Les courants d'air chauds agrandissent les porches lors des canicules et sculptent des alvéoles sur les parois (photo 2).

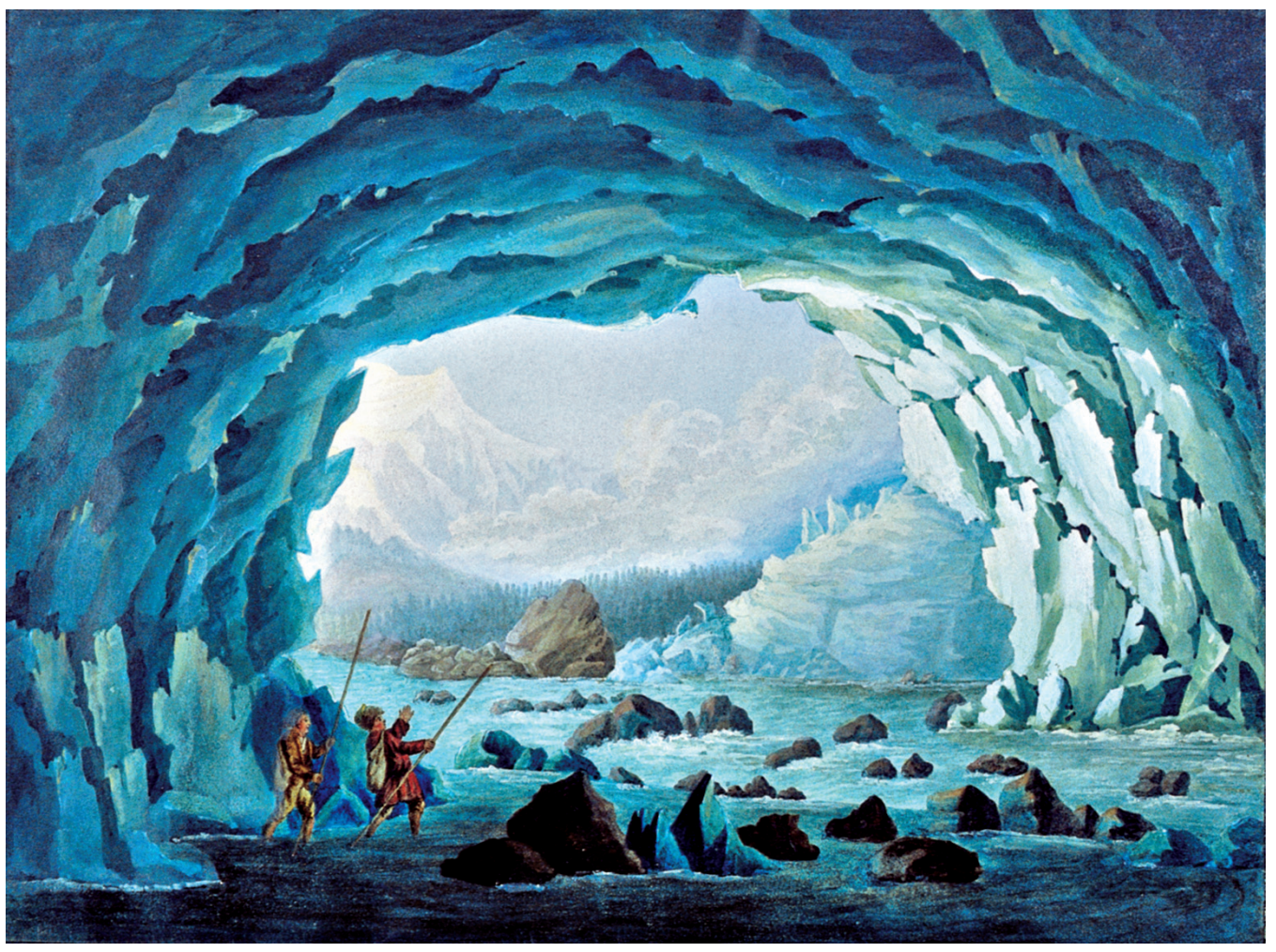

Figure 1 - Visite du porche glaciaire de la Mer de Glace au XVIII ${ }^{e}$ siècle (gravure de Grundman-Lamy, Musée Alpin de Chamonix).

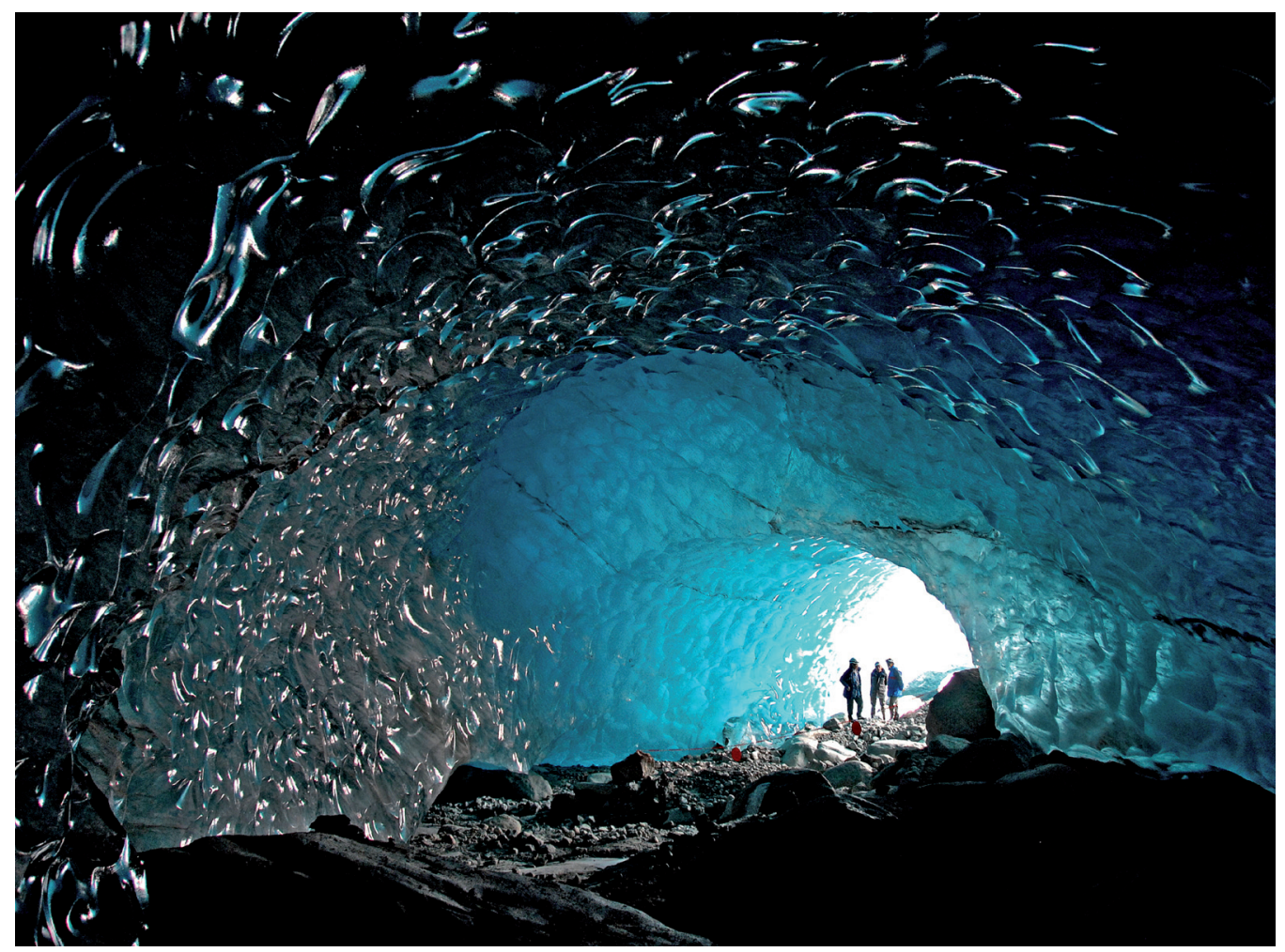

Photo 2 - Portail glaciaire de la Mer de Glace (2008). 
Certains porches glaciaires au Groenland ont permis de remonter plus d'un kilomètre en amont dans le glacier, comme lors de l'expédition 1989 de J. Lamberton et J.-M. Boivin au Groenland occidental. Lorsque la géothermie est active, avec exsurgence d'eaux chaudes, des chenaux parfaitement semi-circulaires sont sculptés et conservés sous la glace toute l'année, comme en Islande sous le Vatnajökull.

Dans ces exutoires naturels, des mesures de l'érosion par les eaux généralement sous pression sont possibles, en encastrant pendant l'hiver dans le lit rocheux des blocs rocheux calibrés. Cette érosion peut atteindre plusieurs millimètres par an sur des gneiss (Röthlisberger, 1972) ou sur des quartzites lorsque les eaux sont chargées de silice (glacier d'Argentière ; Vivian 1975).

\section{2 - Les cavités de décollement sous-glaciaires}

Les cavités sous-glaciaires qui se forment à l'occasion du décollement d'un glacier tempéré à l'aval de protubérances rocheuses sont parfois accessibles sur ses marges, ce qui permet d'y observer la structure de la glace basale, voire de mesurer le glissement basal. Cette glace basale, généralement chargée en sables et particules plus grossières, engendre le polissage du lit rocheux par abrasion. Son épaisseur est variable, mais elle présente une limite franche avec la glace pure au-dessus ; elle résulte du phénomène de fusion-regel lorsque le glacier franchit une protubérance rocheuse : la glace au contact de la face amont de la protubérance fond, du fait de la pression ; cette eau regèle à l'aval, incluant les débris rocheux. L'analyse de son épaisseur et de sa déformation renseigne sur les conditions du glissement et les fortes pressions à la base du glacier.

\section{3 - L'accès est-il possible par les crevasses ?}

Louis Agassiz, qui avait compris l'intérêt d'accéder à l'intérieur du glacier, fût le premier scientifique connu à sonder une crevasse (Agassiz, 1847). Il se fit descendre dans l'une d'elles au bout d'une corde en 1840 - mais celle-ci était remplie d'eau à quelques mètres de profondeur. C'est la volonté de comprendre ce qui gouvernait le mouvement des glaciers, bien visible en surface par l'ouverture des crevasses

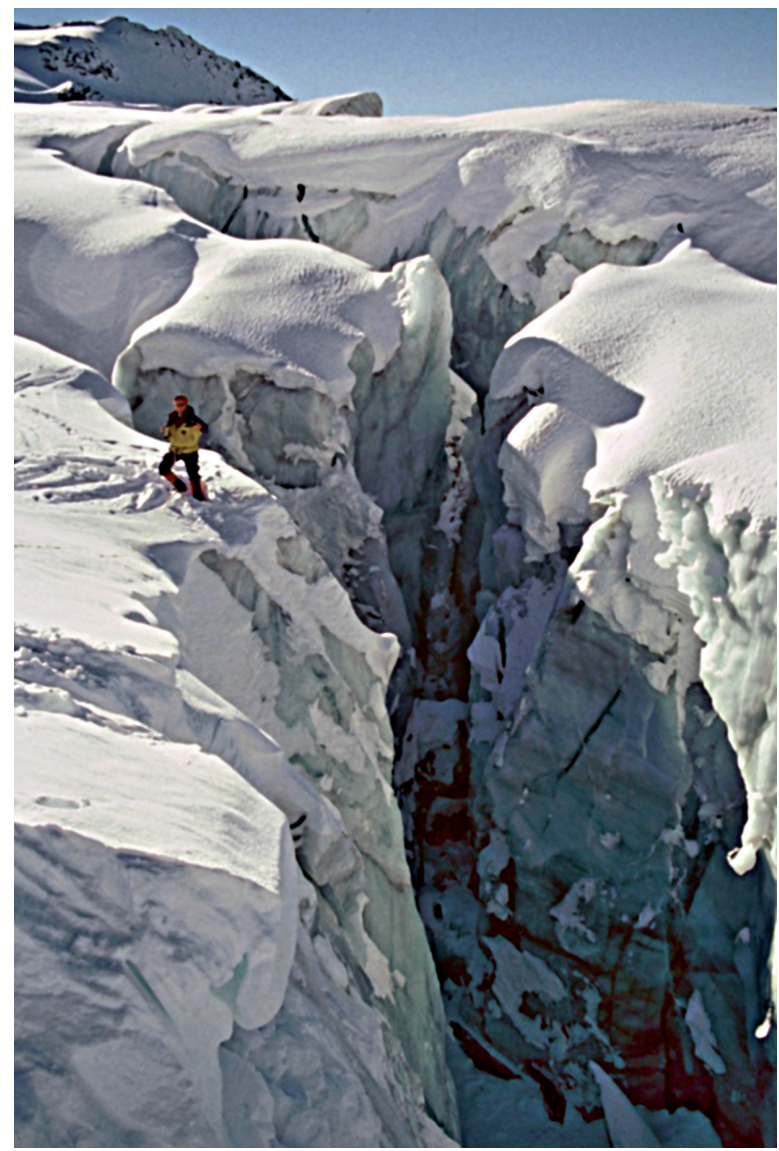

Photo 3 - Crevasse dans le massif du Mont Blanc.

et le déplacement des blocs erratiques comme ceux de l'hôtel des Neuchâtelois sur l'Unteraargletscher, qui explique ces premières tentatives d'observation de l'intérieur au XIX ${ }^{\mathrm{e}}$ siècle.

Les crevasses sont des fractures de surface (photo 3 ) qui résultent de contraintes que la déformation de la glace visco-plastique ne parvient plus à encaisser. Ces contraintes sont engendrées par la pente du glacier, la topographie du lit rocheux, ou la vitesse différente des courants de glace d'un glacier. Les crevasses n'excédent généralement pas 30-35 $\mathrm{m}$ de profondeur dans la glace tempérée, car la déformation en profondeur tend à resserrer les parois. Elles peuvent être plus profondes du fait d'une accumulation de neige en surface, ou dans le cas de la rimaye, qui sépare le glacier de la paroi rocheuse. Si leur exploration est généralement limitée, les crevasses sont aussi à l'origine de puits profonds creusés par l'eau de fonte, les moulins.

\section{II - LES BÉDIÈRES ET LES MOULINS : LE DRAINAGE DES EAUX DE SURFACE}

\section{1 - Les bédières}

Les bédières sont principalement alimentées par les eaux de fonte et, secondairement, par les pluies. Les plus forts débits s'observent en fin d'après midi - des eaux qui ne parviennent à la base du glacier que plu- sieurs heures plus tard. Leur mesure est indispensable si l'on veut évaluer la vitesse de formation des cavités et des moulins (photo 4). Les bédières les plus importantes se trouvent dans les secteurs de fonte étendus et peu pentus - un glacier raide et crevassé ne présente pas de bédières développées. 


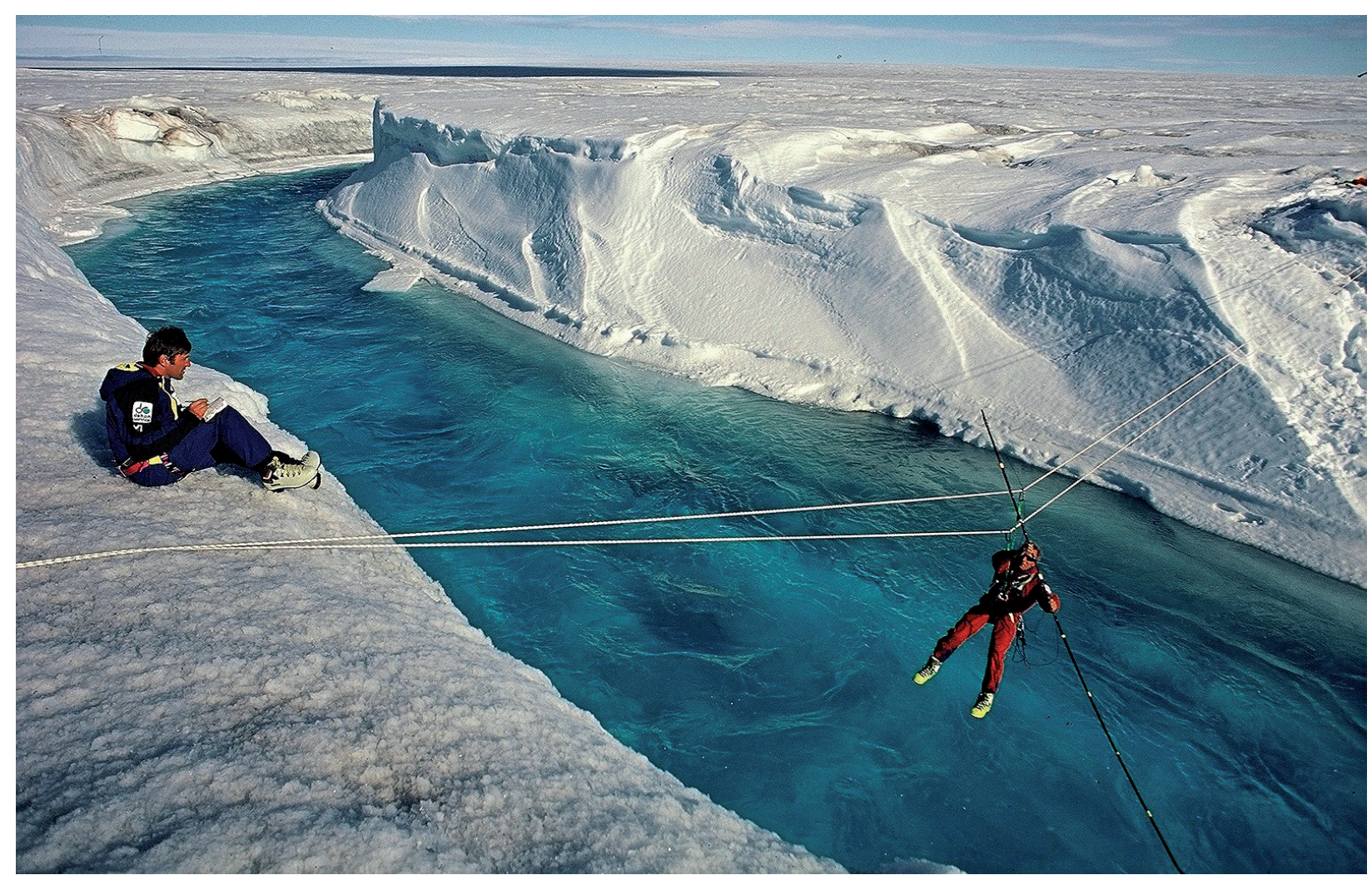

Photo 4 - Mesure de débit sur une bédière au Groenland (photo : P. Bourseiller).

Les bédières de la calotte groenlandaise, bien visibles sur les images satellite, drainent des dizaines de $\mathrm{km}^{2}$ et peuvent atteindre 30 à $50 \mathrm{~km}$ de longueur. Elles apparaissent l'été dès $1500 \mathrm{~m}$ d'altitude (à la latitude du cercle polaire arctique), et s'écoulent vers les marges de l'inlandsis par de grandes surfaces légèrement vallonnées. Leur débit atteint couramment $40-50 \mathrm{~m}^{3} / \mathrm{s}$ (photo 4). Elles ont la particularité d'être souvent couvertes de ponts de neige et de glace, qui se consolident par les embruns qui regèlent par-dessous.

Les bédières permettent en surface des opérations de traçage afin d'évaluer le temps de transfert des eaux de surface vers le front du glacier, avec identification d'une éventuelle rétention (poche d'eau intraglaciaire). Joseph Vallot avait ainsi mesuré un temps de parcours de 2 h 30 depuis le torrent du Nant Blanc en rive droite jusqu'au front de la Mer de Glace, situé à plus de $2 \mathrm{~km}$ en aval (Vallot, 1898).

\section{2 - Les moulins glaciaires : voyage au centre des glaciers}

Les moulins, creusés par l'eau de fonte et présents sur tous les glaciers tempérés, sont un élément essentiel du cryokarst. Ces galeries intraglaciaires subverticales permettent à l'eau qui s'est accumulée dans les crevasses au printemps, sur le fond desquelles elle exerce une forte pression, de se vidanger vers les marges du glacier, ou vers sa base lorsque il est peu épais. Elles sont appelées moulins par analogie avec les anciens moulins à farine alimentés par des dérivations nommées bédières en Savoie - un terme qui dérive de bief ou béal.

En septembre 1897, Vallot explore avec son ami Fontaine le Grand moulin de la Mer de Glace, dans le but d'atteindre le fond du glacier et d'en mesurer l'épaisseur (figure 2); ils descendent à une profondeur de $60 \mathrm{~m}$ avec des échelles de corde de chanvre. D'autres auteurs avaient décrit ces gouffres de glace avant lui, tels Forbes (1843) ou Stoppani en 1876. Depuis Vallot, de nombreuses équipes ont exploré des moulins, notamment des spéléologues dont les techniques et le

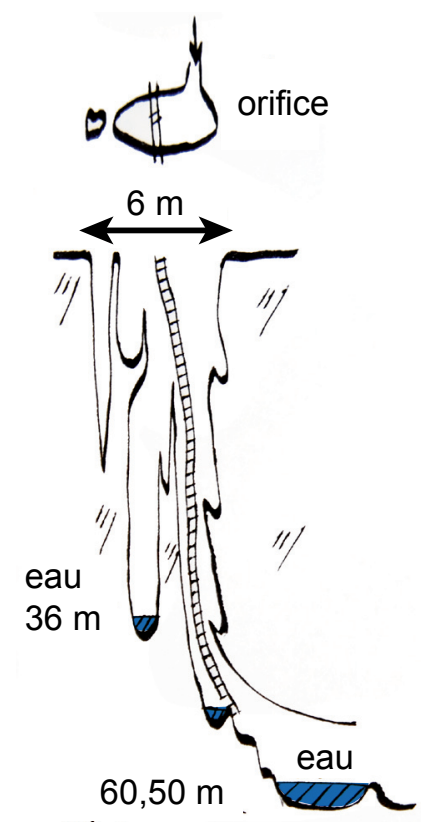

Figure 2 - Coupe du Grand moulin de la Mer de Glace en septembre 1897, d'après J. Vallot. 
matériel sont parfaitement adaptés à ce type d'exploration (photos 5). Mais dans les dizaines de moulins explorés (Groenland, Spitzberg, Baffin, Alpes), malgré des débits parfois élevés et une profondeur maximale de $203 \mathrm{~m}$ au Groenland (pour une épaisseur de glace de $1200 \mathrm{~m}$ ), jamais le substratum rocheux n'a été atteint - sauf là où de faibles épaisseurs et vitesses d'écoulement du glacier se combinent pour permettre à la bédière de creuser chaque année un peu plus profondément le même moulin, comme au glacier Loven est (Spitzberg). En effet, comme les crevasses, les moulins se forment toujours aux mêmes endroits : s'ils se déplacent bien avec la glace, ils sont remplacés après une ou plusieurs années par un nouveau moulin, comme pour le Grand moulin de la Mer de Glace, qui fonctionne deux ou trois étés consécutifs. Les différentes explora-
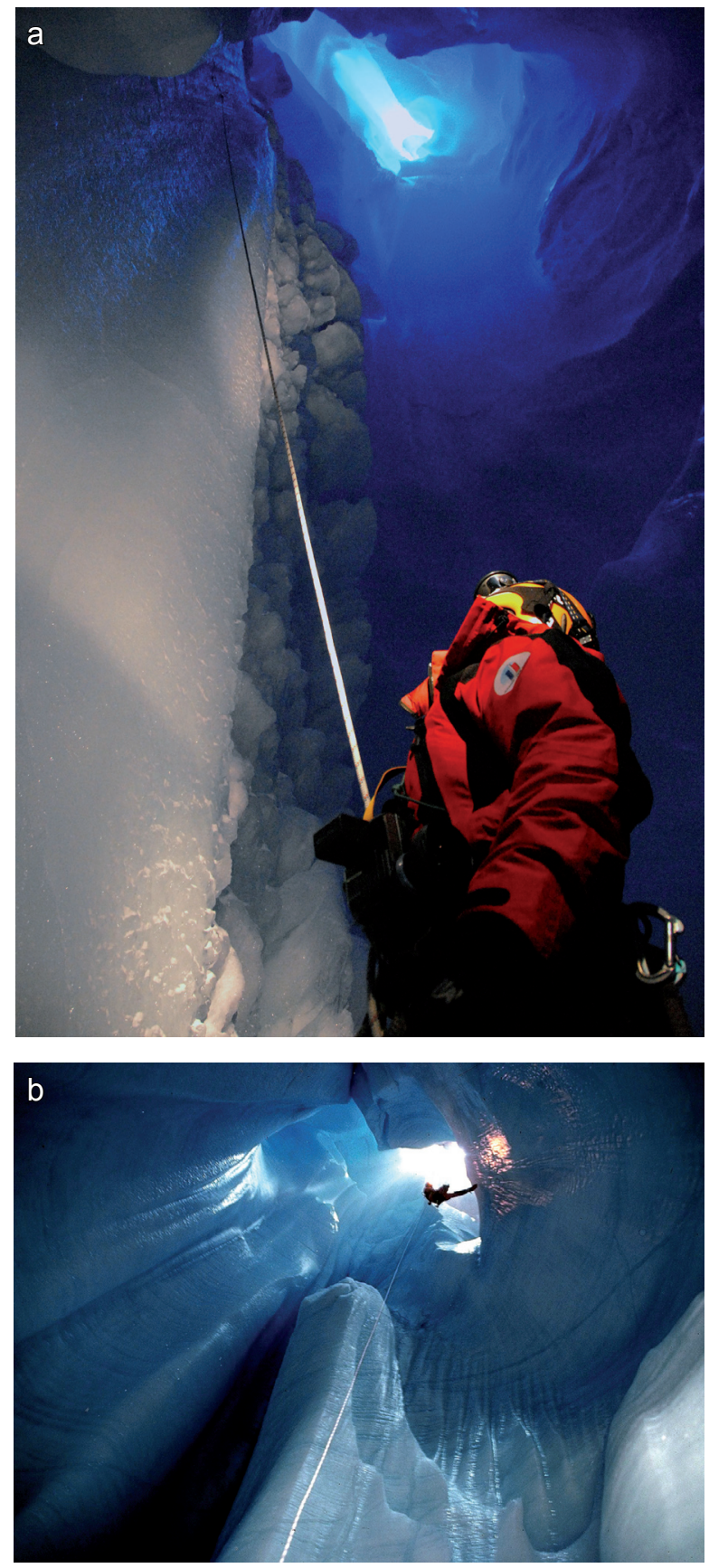

tions de celui-ci (Vallot et Fontaine en 1897, Reynaud en 1986 et 1988, Moreau en 1994 et 1995) montrent des caractéristiques constantes : un puits principal de 4 à $10 \mathrm{~m}$ de diamètre et 60 à $70 \mathrm{~m}$ de profondeur, puis une galerie d'évacuation en gradins, sinueuse (Figure 3). Les explorations entre 1986 et 1994 n'ont pu descendre au-delà de 95-110 $\mathrm{m}$ de profondeur et suivre le cheminement de l'eau (Moreau, 1995). Mais le glacier très épais $(350 \mathrm{~m})$ ne devrait pas pouvoir conserver de conduits sous-glaciaires à la base du glacier, du fait de la pression et de la déformation. L'eau de fonte ne doit accéder à l'interface glace-roche que lorsque l'épaisseur du glacier n'est pas trop importante (probablement $<150 \mathrm{~m}$ ). Toutefois, lorsque le lit rocheux présente une forte rugosité (protubérances, barres rocheuses), l'eau doit pouvoir circuler sous le glacier malgré une épaisseur plus importante.

De nombreux auteurs considèrent que l'augmentation de la vitesse des glaciers en été résulte d'une augmentation du débit des eaux de fonte à leur base, ce que confirment nos mesures effectuées en continu sous le glacier d'Argentière (figure 4). En revanche, l'interprétation par certains auteurs d'anomalies verticales sur des images radar de l'inlandsis groenlandais (Catania et al., 2008) est étonnante : elles résulteraient de la circulation d'eau dans les moulins sous plus de $1000 \mathrm{~m}$ de glace !

Dans le cas de la Mer de Glace, l'eau du Grand moulin rejoint la rive droite, comme le suggèrent les conduits orientés vers le nord et les conduits fossiles qui apparaissent quelques années plus tard à la surface du glacier à l'aval du moulin, du fait de l'ablation glaciaire (photo 6). Si le tracé de la connexion avec la base du glacier doit être en partie contrôlé par les fractures internes, aucune étude n'a jusqu'à présent démontré cette connexion.

Les moulins permettent également d'observer la structure de la glace en profondeur, comme la foliation ou les bandes de Forbes (photo 7). On peut y mesurer les caractéristiques de la déformation de la glace, et échantillonner sur les parois. La meilleure période pour une telle exploration est celle qui suit immédiatement l'arrêt de la fonte, avant que le moulin ne se referme par déformation, ou quand l'eau de la bédière est capturée par une nouvelle crevasse qui s'ouvre à l'amont. Une situation de ce type nous a permis de mesurer en 1996 au Groenland ouest la vitesse de resserrement des parois dans un moulin actif durant 10 jours, à l'aide d'un télémètre SONIN (Bourseiller et al., 2002). Un réseau de 10 balises en bois ancrées dans les parois a été installé dans le moulin Louisa, à $120 \mathrm{~m}$ de profondeur et à $3 \mathrm{~m}$ au-dessus du fond du moulin; il a mesuré un resserrement de $69 \mathrm{~cm}$ selon le plus grand axe, avec une température de la glace de $-1,3{ }^{\circ} \mathrm{C}$ (tableau 1).

Photos 5 - Moulins au Groenland.

$a:-140 \mathrm{~m}$ (Expédition 2007);

b :-120 m (Expédition 1993 ; photo : J.-P. Astruc). 

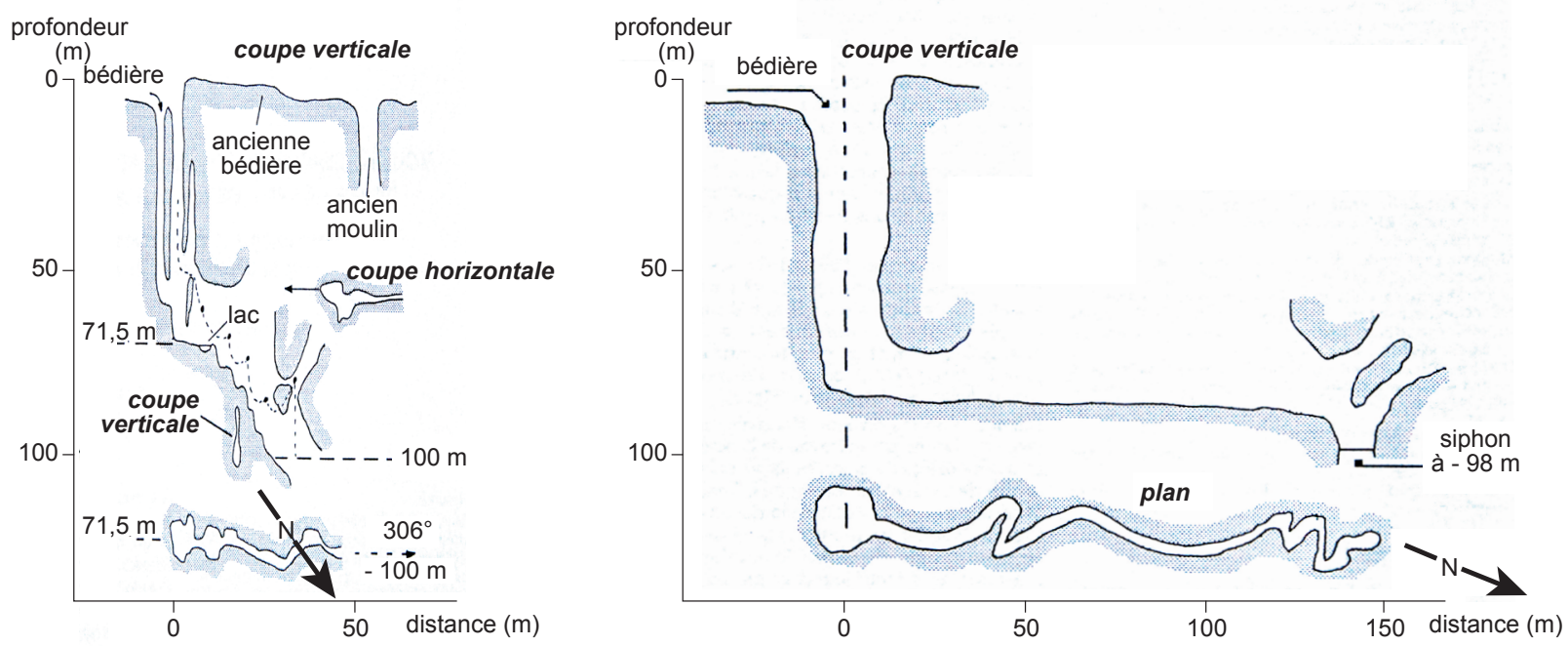

Figure 3 - Coupes du Grand moulin de la Mer de Glace en septembre1986 (à gauche) et 1988 (à droite), d'après L. Reynaud.

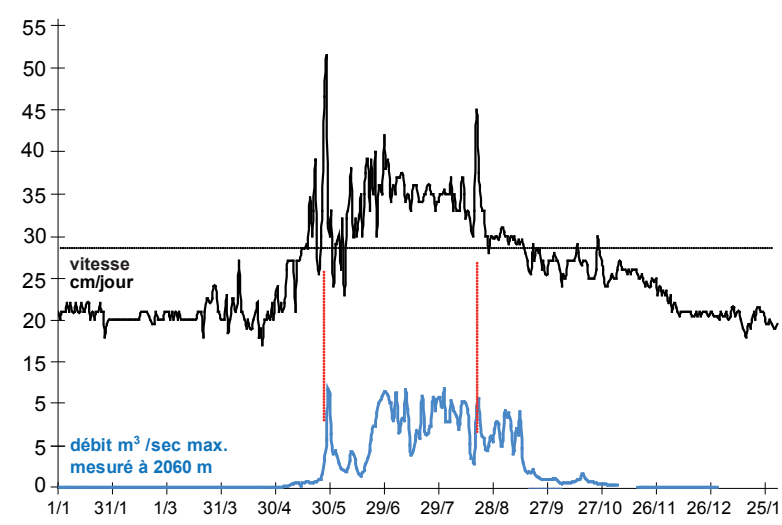

Figure 4 - Débit et vitesse de glissement du glacier d'Argentière en 2008 (données : Emosson SA et L. Moreau).

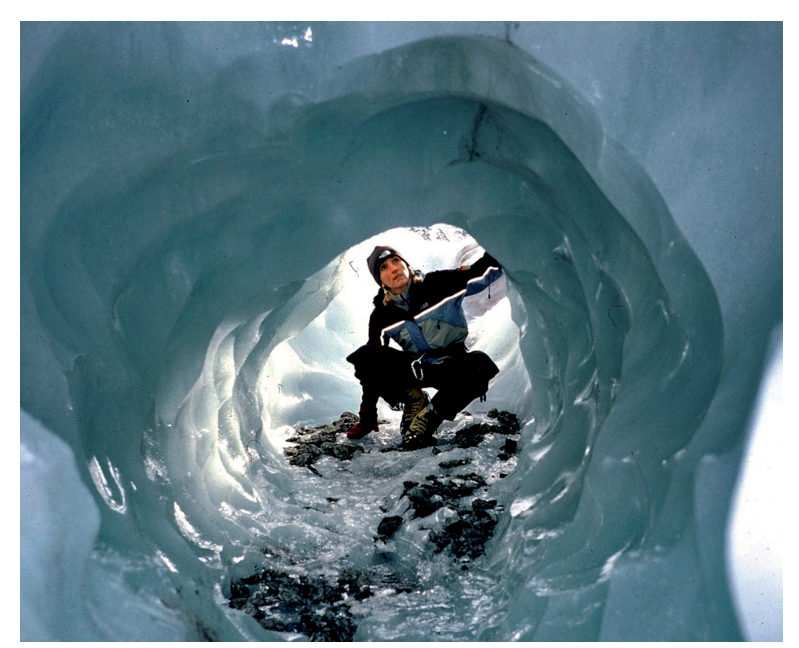

Photo 6 - Conduit intraglaciaire fossile, parvenu à la surface de la Mer de Glace du fait de l'ablation glaciaire.

\begin{tabular}{|ccccccc|}
\hline Date & $04 / 09$ & $06 / 09$ & $08 / 09$ & $10 / 09$ & $15 / 09$ & $16 / 09$ \\
\hline $\begin{array}{c}\text { Largeur } \\
\text { galerie }(\mathrm{cm})\end{array}$ & 202 & 200 & 192 & 160 & 134 & 133 \\
\hline
\end{tabular}

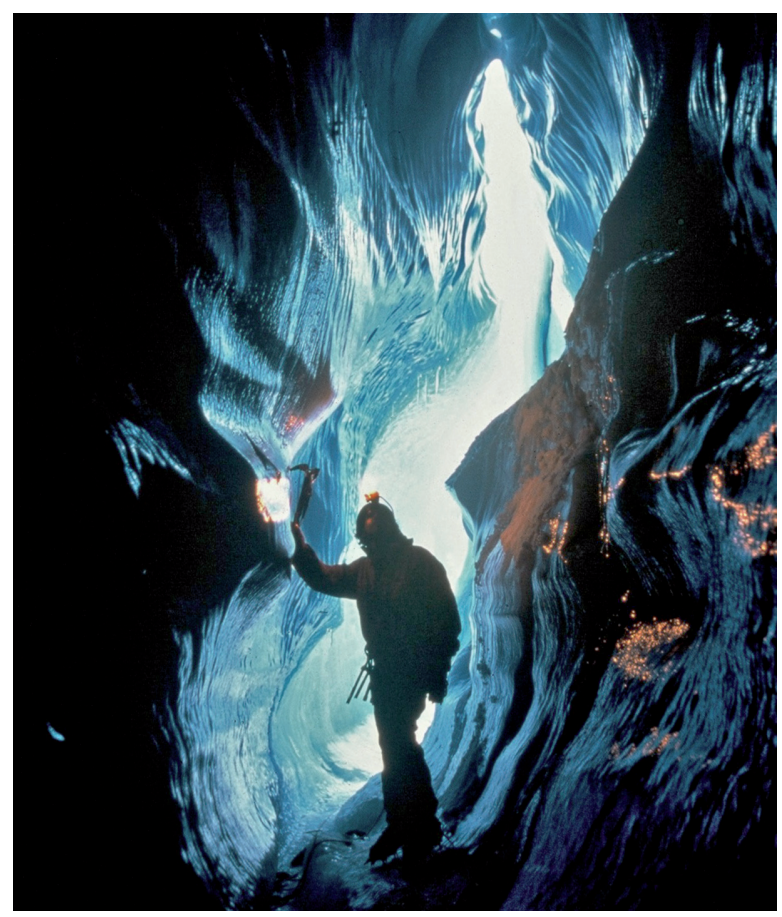

Photo 7 - Conduit intraglaciaire prolongeant le Grand moulin de la Mer de Glace (photo : J.-F. Hagenmüller).

L'automne et l'hiver, lorsque les débits diminuent, la déformation de la glace devient plus rapide que son creusement. De ce fait, les moulins se referment en profondeur et, bien souvent, se remplissent d'eau. Comme en témoignent les corniches de glace columnaire de regel accrochées sur les parois du Grand moulin de la Mer de Glace ou des moulins groenlandais, ces eaux percolent ensuite durant l'hiver à travers le glacier, comme Vallot en avait déjà fait l'observation en 1897.

Notons que la décrue actuelle des glaciers peut être favorable au développement de bédières et de moulins,

Tableau 1 - Vitesse de fermeture d'un moulin à $120 \mathrm{~m}$ de profondeur au Groenland ouest en 1996. 
car le ralentissement du glacier s'accompagne d'une moindre fracturation de surface dans la zone d'ablation, d'où un bassin collecteur plus vaste. Ainsi, on observe depuis 2005 sur la Mer de Glace deux bédières centrales subparallèles sous le Montenvers, à 1700 m d'altitude, qui se terminent par deux moulins, explorés à l'automne 2007 jusqu'à près de $70 \mathrm{~m}$ de profondeur (épaisseur du glacier $>100 \mathrm{~m}$ ).

\section{3 - Circulation latérale d'eau sous-glaciaire et érosion des auges glaciaires}

Lorsqu'une bédière est recoupée par une crevasse marginale sur un glacier alpin, ses eaux sont dirigées vers les marges rocheuses encaissantes (figure 5). Cette circulation latérale des eaux, sous pression et à vitesse élevée, est à l'origine d'une action érosive intense, car ces eaux sont chargées de graviers et sables. Elle contribue probablement à l'élargissement de l'auge glaciaire, comme le suggèrent les marmites de géant glaciaires

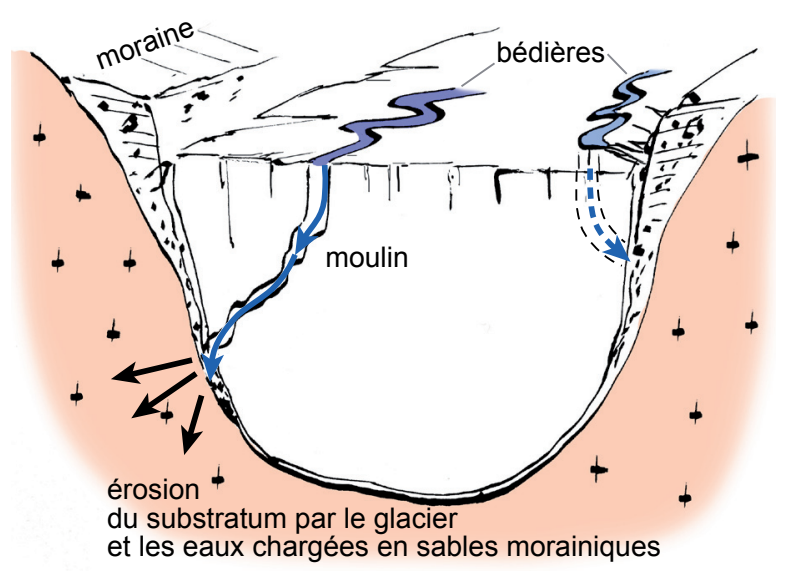

Figure 5 - Drainage interne latéral observé à la mer de glace et érosion sur les parois de l'auge glaciaire (dessin L. Moreau).

que l'on peut trouver à mi-hauteur des parois rocheuses dans les vallées déglacées, comme par exemple à Servoz, dans la vallée de l'Arve - un processus qui n'a pas toutefois été étudié à notre connaissance.

\section{Conclusion}

Les paramètres hydrologiques sous-glaciaires qui influencent l'écoulement des glaciers tempérés ou froids restent encore mal connus du fait des difficultés d'accès, même si l'exploration des moulins depuis une quarantaine d'années a permis d'en améliorer les connaissances. Les observations en profondeur ont ainsi montré que les eaux s'écoulent souvent vers les marges $\mathrm{du}$ glacier de vallée, faute d'accès au fond rocheux, tandis qu'il a été possible de mesurer les variations de la circulation d'eau et sa contribution à l'érosion de la glace et du substratum rocheux, sous le glacier comme sur ses marges.

Il reste à comprendre en particulier comment les eaux parviennent à l'interface glacier-lit rocheux. Cette prochaine étape scientifique d'exploration permettra de compléter la compréhension de l'écoulement des glaciers, avec en particulier l'accélération actuelle des glaciers polaires (influence sur le niveau de l'océan à court terme), ou les accélérations brutales (surges) de certains glaciers. Or, du fait du changement climatique très rapide en cours dans les régions polaires, ces dynamiques pourraient être plus fréquentes. Enfin, les glaciers alpins froids, soudés jusqu'à aujourd'hui au rocher, verront leurs conditions d'écoulement fortement modifiées lorsque leur glace basale atteindra le point de fusion.

\section{Remerciements}

À Sylvain Coutterand pour l'aide fournie sur la mise en forme des croquis.

\section{BiBLIOGRAPHIE}

Agassiz L., 1847. Système glaciaire ou recherches sur les glaciers. $1^{\text {ière }}$ partie : Nouvelles études et expériences sur les glaciers actuels, leur structure, leur progression et leur action physique sur le sol. Masson, Paris, XXXI +598 p.

Bourseiller P., Lamberton J., Couté A., Moreau L., 2002. Voyage dans les glaces. La Martinière, Paris, 195 p.

Catania, G., Neumann, T., Price, S., 2008, Characterizing englacial drainage in the ablation zone of the Greenland ice sheet. Journal of Glaciology, 54 (187), 567-578.

ForBes J.D., 1843. Travels through the Alps of Savoy and others parts of the Pennine chain. Black, Edinburgh, $460 \mathrm{p}$.
Moreau L., 1995. Moulins glaciaires des glaciers tempérés et froids de 1986 à 1994 (Mer de Glace et Groenland), morphologie et techniques de mesure de la déformation de la glace, Actes du $3^{\text {e }}$ symposium international Cavités glaciaires et cryokarst en régions polaires et de haute montagne, Chamonix, novembre 1994, 109-113.

Reynaud L., 1987. The November 86 survey of the Grand Moulin on the Mer de Glace, Mont-Blanc. Journal of Glaciology, 33, 113, 130-131.

RothLisberger H., 1972. Water pressure in intra and subglacial channels. Journal of Glaciology, 11, 62, 205-214.

VALLOT J., 1898. Explorations des moulins de la mer de glace. Annales de l'Observatoire du Mont-Blanc, 3, 183-190.

Vivian R., 1975. Les glaciers des Alpes occidentales. Allier, Grenoble, $513 \mathrm{p}$. 



\section{NEIGE et GLACE de MONTAGNE \\ Reconstitution, dynamique, pratiques}

\section{Sommaire}

Editorial

Introduction

\section{1 - Reconstitution}

Coutterand S. et al. - Le lobe glaciaire lyonnais au maximum würmien : glacier du Rhône ou/et glaciers savoyards ?

Ravanel L. et al. - Désenglacement du haut bassin versant du Vorz (massif de Belledonne, Isère), au Tardiglaciaire et à l'Holocène.

Rey P.-J. - Sociétés et fluctuations du climat dans les Alpes nord-occidentales au Néolithique moyen.

Le Roy et al. - Étude des fluctuations glaciaires du Petit Âge de Glace dans le Massif des Écrins : apports de la lichénometrie.

Kirkbride M.P. - Datation des moraines holocènes d'Islande par tephrochronologie : un état de l'art.

Le Roy et al. - La dendroglaciologie, ou l'apport de l'étude des cernes d'arbres pour la reconstitution des fluctuations glaciaires holocènes.

Rabatel A. - Évolution glaciaire dans les andes subtropicales chiliennes entre 1955 et 2007 : conséquences pour la ressource en eau.

Le Roy et al. - Un inventaire des aérophotographies du massif du Mont Blanc.

\section{2 - Dynamique}

Ravanel L. - Évolution géomorphologique de la haute montagne alpine dans le contexte actuel de réchauffement climatique.

Gruber S. - Le permafrost de haute montagne.

Deline P. et al. - L'Aiguille du Midi (massif du Mont Blanc) : un site remarquable pour l'étude du permafrost des parois d'altitude.

Saulnier G.-M. et al. - Un éléphant volant est-il un oiseau ? Perspectives pour l'observation hydrométéorologique des milieux de montagne.

Jobard S. - L'instrumentation du glacier du Baounet : quels apports pour la traçabilité des mesures environnementales ?

Moreau L. - L'exploration du cryokarst glaciaire et son intérêt scientifique pour l'étude du drainage des eaux de fonte.

Mazué R. et al. - Suivi de l'évolution de la couverture detritique d'un glacier noir par photo-comparaison : le glacier d'Estelette.

\section{3 - Pratiques}

Paccard P. - Réchauffement climatique et ressource neige en domaines skiables.

Gauchon C. - Les hivers sans neige et l'économie des sports d'hiver : un phénomène récurrent, une problématique toujours renouvelée.

Laslaz L. - L'exclusion des glaciers des zones centrales des Parcs nationaux de la Vanoise et des Écrins et leur équipement pour le ski d'été.

Cayla N. - Les sentiers d'interprétation glaciaire : des outils de valorisation différenciée des glaciers et de leur territoire.

Lambert R. - Cartozonage : de la carte au zonage du risque avalanche.

Moulin A. et al. - L'incertitude liée aux avalanches dans les Alpes du Nord : identifications et implications pour la gestion.

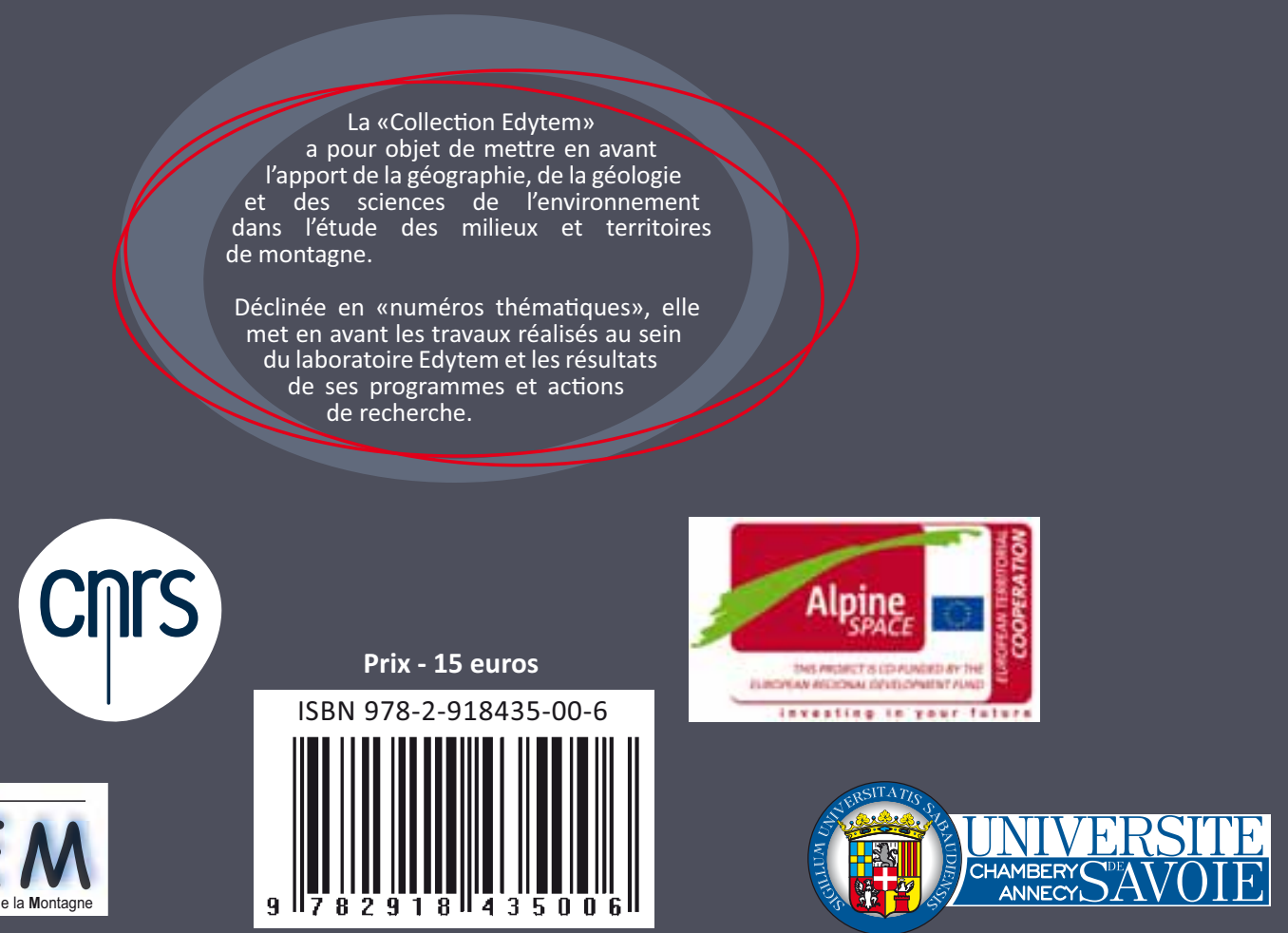

